\title{
Suppressive Effect of Insulin on the Gene Expression and Plasma Concentrations of Mediators of Asthmatic Inflammation
}

\author{
Husam Ghanim, ${ }^{1}$ Kelly Green, ${ }^{1}$ Sanaa Abuaysheh, ${ }^{1}$ Manav Batra, ${ }^{1}$ \\ Nitesh D. Kuhadiya, ${ }^{1}$ Reema Patel, ${ }^{1}$ Antoine Makdissi, ${ }^{1}$ Sandeep Dhindsa, ${ }^{2}$ \\ Ajay Chaudhuri, ${ }^{1}$ and Paresh Dandona ${ }^{1}$ \\ ${ }^{1}$ Division of Endocrinology, Diabetes and Metabolism, State University of New York at Buffalo and Kaleida Health, \\ 115 Flint Road, Williamsville, NY 14221, USA \\ ${ }^{2}$ Division of Endocrinology and Metabolism, Texas Tech University Health Sciences Center, 701 W 5th Street, Odessa, TX 79763, USA
}

Correspondence should be addressed to Paresh Dandona; pdandona@kaleidahealth.org

Received 28 August 2014; Revised 20 November 2014; Accepted 18 December 2014

Academic Editor: Francis M. Finucane

Copyright (C) 2015 Husam Ghanim et al. This is an open access article distributed under the Creative Commons Attribution License, which permits unrestricted use, distribution, and reproduction in any medium, provided the original work is properly cited.

\begin{abstract}
Background and Hypothesis. Following our recent demonstration that the chronic inflammatory and insulin resistant state of obesity is associated with an increase in the expression of mediators known to contribute to the pathogenesis of asthma and that weight loss after gastric bypass surgery results in the reduction of these genes, we have now hypothesized that insulin suppresses the cellular expression and plasma concentrations of these mediators. Methods. The expression of IL-4, LIGHT, LTBR, ADAM-33, and TSLP in MNC and plasma concentrations of LIGHT, TGF- $\beta 1$, MMP-9, MCP-1, TSLP, and NOM in obese patients with T2DM were measured before, during, and after the infusion of a low dose ( $2 \mathrm{U} / \mathrm{h})$ infusion of insulin for 4 hours. The patients were also infused with dextrose or saline for 4 hours on two separate visits and served as controls. Results. Following insulin infusion, the mRNA expression of IL-4, ADAM-33, LIGHT, and LTBR mRNA expression fell significantly $(P<0.05$ for all). There was also a concomitant reduction in plasma NOM, LIGHT, TGF- $\beta 1$, MCP-1, and MMP-9 concentrations. Conclusions. Insulin suppresses the expression of these genes and mediators related to asthma and may, therefore, have a potential role in the treatment of asthma.
\end{abstract}

\section{Introduction}

We have recently demonstrated that the chronic inflammatory states of obesity and type 2 diabetes are associated with an increase in the cellular expression and the plasma concentrations of mediators involved in the pathogenesis of asthma, consistent with the increase in the prevalence of asthma in these conditions [1]. These data provide the first mechanistic link between obesity and asthma, beyond the obvious effects on lung volumes. Furthermore, our data provided the first evidence that, following gastric bypass surgery and weight loss, there was a significant reduction in these mediators including IL-4, the key TH-2 cytokine associated with asthma [2].

Other inflammatory mediators, which play a role in asthma, include matrix metalloproteinases (MMP), including MMP-9 and ADAM-33 (a disintegrin and metalloproteinase$33)$; chemokines including eotaxins, RANTES, and MCP1; chemokine receptors, CCR-3 for eotaxin, CCR-5 for
RANTES, and CCR-2 for MCP-1 [3, 4]. More recently, it has been shown that LIGHT and its receptor, lymphotoxin$\beta$ receptor (LTBR), also participate in asthmatic inflammation by mediating remodeling of bronchi and bronchioles. The remodeling action of LIGHT-LTBR is mediated by TGF $\beta$ which induces fibrosis and also leads to epithelialmesenchymal transformation [5]. Most recently, thymic stromal lymphopoietin (TSLP) has been shown to contribute to the pathogenesis of asthma and treatment of asthmatic patients with a monoclonal antibody directed against TSLP improves respiratory function [6]. In addition to the possible contribution of these proinflammatory mediators, there is also an increase in nitric oxide (NO) and isoprostane content in exhaled air in asthmatics when compared to normal subjects [7]. The increase in NO is likely due to the activation of iNOS in bronchial macrophages and is thought to relate to clinical activity of asthma [8]. Increased isoprostane generation is a reflection of increased oxidative stress which also 
characterizes asthmatic inflammation [9]. We have recently shown that obesity and diabetes are associated with an increase in the expression of IL-4, LIGHT, CCR-2, and MMP9 in $\mathrm{MNC}$ and that, following gastric bypass surgery, there is a reduction in the expression of these genes [1]. In addition, there is also a reduction in the expression of ADAM-33 in parallel with weight loss and an increase in insulin sensitivity.

Our previous work has shown that insulin exerts a potent and rapid anti-inflammatory effect inducing a suppression of major transcription factors like $\mathrm{NF} \kappa \mathrm{B}$ and Egr-1 along with a reduction in the concentration of several proinflammatory mediators in plasma including ICAM-1, MCP-1, MMP-9, PAI-1, and VEGF and the expression of inflammatory cytokines and chemokines in the MNC [10-12]. More recently, a low dose insulin infusion has also been shown to suppress the expression of several toll-like receptors including TLRs 1, 2, 4, 7, and 9; PU.1 DNA binding; and TLR2 protein in MNC from obese patients with T2DM [13]. PU.1 is the major transcription factor modulating the transcription of TLRs. The suppression of TLRs is important in the context of asthma since TLRs are pathogen recognition receptors and bacterial and viral infections are important triggers in the exacerbation of inflammation in asthma [14].

On the basis of the above, we hypothesized that the expression of IL-4, ADAM-33, MMP-9, LIGHT, LTBR, and TSLP and the plasma concentration of LIGHT, NOM, MCP-1, MMP-9, TGF- $\beta$, and TSLP are suppressed by an intravenous infusion of insulin. These factors/genes have mostly been associated with asthma during the past decade and, therefore, were specifically included in our hypothesis. In addition, these are the genes we investigated in our previous paper based on data from morbidly obese patients before and after gastric bypass surgery [1].

\section{Methods}

\subsection{Subjects}

2.1.1. Insulin Infusion Study. Ten obese patients with type 2 diabetes (T2DM) ( 5 females and 5 males, age: $47.9 \pm 8.9$ years; BMI: $39.2 \pm 6.5 \mathrm{~kg} / \mathrm{m}^{2}$ ) were recruited for a crossover insulin infusion study. The diabetics had a mean HbAlc of $7.0 \pm$ $0.81 \%$. They were on stable oral antidiabetic medications. All patients were on metformin (1-2 g/day) and 6 patients were on sulfonylureas (glyburide or glipizide 5-10 mg/day). After an overnight fast, subjects were infused with insulin $(2 \mathrm{U} / \mathrm{h})$ with $5 \%$ glucose $(100 \mathrm{~mL} / \mathrm{h})$ and $20 \mathrm{mEq}$ of potassium chloride per hour for $4 \mathrm{~h}$ followed by $2 \mathrm{~h}$ of observation. Blood glucose levels were measured every 15 minutes and were maintained at a target level of $80-130 \mathrm{mg} / \mathrm{dL}$. On a separate visit, all subjects were also infused with $5 \%$ glucose alone at a rate of $100 \mathrm{~mL} / \mathrm{h}$ to control for the dextrose infused with insulin. On the third visit, they were infused with normal saline alone at a rate of $100 \mathrm{~mL} / \mathrm{h}$ for $4 \mathrm{~h}$ to control for the volume infused during the insulin or glucose infusions. Since four patients declined participation in the normal saline arm of the study, only 6 patients ( 4 females and 2 males, age: $41.5 \pm 8.2$ years, BMI $36.9 \pm 6.7 \mathrm{~kg} / \mathrm{m}^{2}$, and HbAlc of $7.5 \pm 1.1 \%$ ) were infused with saline. Blood samples were collected at baseline and at
$2 \mathrm{~h}, 4 \mathrm{~h}$, and $6 \mathrm{~h}$ following the infusion. The protocol was approved by the Human Research Committee of the State University of New York at Buffalo. An informed consent was signed by all subjects.

2.2. MNC Isolation. Blood samples were collected in $\mathrm{Na}-$ EDTA and carefully layered on Lympholyte medium (Cedarlane Laboratories, Hornby, ON). Samples were centrifuged and two bands separated out at the top of the RBC pellet. The MNC band was harvested and washed twice with Hank's balanced salt solution (HBSS). This method provides yields greater than $95 \%$ MNC preparation.

2.3. ROS Generation Measurement by Chemiluminescence. Five hundred $\mu \mathrm{L}$ of MNC $\left(2 \times 10^{5}\right.$ cells $)$ was delivered into a cuvette. Luminol was then added, followed by $1.0 \mu \mathrm{L}$ of $10 \mathrm{mM}$ formylmethionyl leucyl phenylalanine (fMLP) and chemiluminescence was recorded for 5 min using Chronolog Lumi-Aggregometer as previously described [15].

2.4. Quantification of $m R N A$ in MNC by RT-PCR. Total RNA was isolated using commercially available RNAqueous-4PCR Kit (Ambion, Austin, TX). RT-PCR was performed using Cepheid Smart Cycler (Sunnyvale, CA), Sybergreen Master mix (Qiagen, CA), and gene specific primers for IL-4, LIGHT, LTBR, TSLP, and ADAM-33 (Life Technologies, MD). All samples were assayed for a group of 4 housekeeping genes: $\beta$-actin, ubiquitin C, cyclophilin A, and RPS3 (Biosearch Technologies, Inc., Petaluma, CA). A normalization factor based on the values of all housekeeping genes for each sample was calculated by GeNorm software (Qbase) and was used to normalize the expression of the genes of interest.

2.5. Plasma Measurements. Glucose concentrations were measured in plasma by YSI 2300 STAT Plus glucose analyzer (Yellow Springs, OH). ELISA was used to measure insulin (Diagnostic Systems Laboratories Inc., Webster, TX), MCP-1, MMP-9, TGF- $\beta 1$, LIGHT (R\&D Systems, Minneapolis, MN), and TSLP (Biolegend, San Diego, CA). Plasma concentrations of nitric oxide metabolites ( $\mathrm{NOM}: \mathrm{NO}_{2} / \mathrm{NO}_{3}$ ) were measured by Griess reaction using a colorimetric assay kit from $\mathrm{R} \& \mathrm{D}$ Systems (Minneapolis, MN).

2.6. Statistical Analysis. Statistical analysis was conducted using SigmaStat software (SPSS Inc., Chicago, IL). All data are represented as mean \pm SE. Statistical analysis from baselines was carried out using Holm-Sidak one-way repeated measures analysis of variance (RMANOVA). Dunnett's twofactor RMANOVA method was used for multiple comparisons between different groups. Demographic variables and baseline levels of inflammatory mediators in lean, obese, and obese T2DM patients were compared using Student's $t$-test. $P<0.05$ was considered significant.

\section{Results}

3.1. Insulin and Glucose Concentrations following Insulin Infusion. Mean blood glucose concentrations did not change 
TABLE 1: Change in inflammatory and oxidative stress mediators in MNC and serum following $2 \mathrm{U} / \mathrm{hr}$ insulin/dextrose infusion (insulin), dextrose alone (dextrose), or saline alone (saline) in obese T2DM for 4 hours. Data are presented as mean \pm SE. ${ }^{*} P<0.05$ by one-way RMANOVA (compared to baseline as absolute value and percent change); ${ }^{*} \mathrm{P}<0.05$ by two-way RMANOVA compared to control groups (as percent change from baseline).

\begin{tabular}{|c|c|c|c|c|c|}
\hline & Group & $0 \mathrm{~h}$ & $2 \mathrm{~h}$ & $4 \mathrm{~h}$ & $6 \mathrm{~h}$ \\
\hline \multirow{3}{*}{ Glucose (mg/dL) } & Saline & $134 \pm 16$ & $118 \pm 14$ & $112 \pm 12$ & $107 \pm 11^{*}$ \\
\hline & Dextrose & $133 \pm 14$ & $135 \pm 11$ & $125 \pm 11$ & $110 \pm 10^{*}$ \\
\hline & Insulin & $122 \pm 15$ & $114 \pm 12$ & $111 \pm 10$ & $109 \pm 12^{*}$ \\
\hline \multirow{3}{*}{ Insulin $(\mu \mathrm{U} / \mathrm{mL})$} & Saline & $24.1 \pm 4$ & $25.4 \pm 6$ & $21.8 \pm 5$ & $22.4 \pm 6$ \\
\hline & Dextrose & $27.6 \pm 5$ & $25.4 \pm 6$ & $22.9 \pm 6$ & $23.5 \pm 7$ \\
\hline & Insulin & $20.1 \pm 3$ & $50.5 \pm 8^{* \#}$ & $43.8 \pm 9^{* \#}$ & $24.8 \pm 4$ \\
\hline \multirow{3}{*}{ MNC ROS generation (\%) } & Saline & 100 & $97 \pm 8$ & $102 \pm 7$ & $96 \pm 9$ \\
\hline & Dextrose & 100 & $109 \pm 7$ & $112 \pm 8$ & $104 \pm 7$ \\
\hline & Insulin & 100 & $95 \pm 6$ & $82 \pm 5^{* \#}$ & $112 \pm 9$ \\
\hline \multirow{3}{*}{ MCP-1 (ng/mL) } & Saline & $275 \pm 36$ & $277 \pm 38$ & $269 \pm 34$ & $262 \pm 34$ \\
\hline & Dextrose & $256 \pm 38$ & $262 \pm 33$ & $258 \pm 31$ & $269 \pm 36$ \\
\hline & Insulin & $270 \pm 40$ & $233 \pm 30^{*}$ & $225 \pm 29^{* \#}$ & $282 \pm 41$ \\
\hline \multirow{3}{*}{ MMP-9 (ng/mL) } & Saline & $354 \pm 58$ & $342 \pm 49$ & $339 \pm 55$ & $341 \pm 47$ \\
\hline & Dextrose & $327 \pm 63$ & $312 \pm 55$ & $313 \pm 57$ & $315 \pm 48$ \\
\hline & Insulin & $367 \pm 58$ & $338 \pm 47$ & $321 \pm 31^{* \#}$ & $352 \pm 44$ \\
\hline
\end{tabular}

significantly during the first 4 hours of the insulin, dextrose, or saline infusions but did fall significantly at $6 \mathrm{hr}$ ( 2 hours following cessation of the infusions) in all groups (Table 1). Blood glucose at baseline and at $4 \mathrm{~h}$ was not significantly different between the three groups. Plasma insulin concentration increased from $20.9 \pm 10.9 \mu \mathrm{U} / \mathrm{mL}$ to $50.5 \pm 22.4 \mu \mathrm{U} / \mathrm{mL}$ $(P<0.001)$ during the insulin infusion while it fell slightly in the dextrose groups from $27.6 \pm 5.6 \mu \mathrm{U} / \mathrm{mL}$ to $22.9 \pm$ $6.5 \mu \mathrm{U} / \mathrm{mL}$ at $4 \mathrm{~h}$ (NS) and in the normal saline group from $20.6 \pm 5.5 \mu \mathrm{U} / \mathrm{mL}$ to $17.9 \pm 4.7 \mu \mathrm{U} / \mathrm{mL}$ at $4 \mathrm{~h}$ (NS) (Table 1 ).

\subsection{Effect of Insulin Infusion on Expression of IL-4, ADAM-33,} LIGHT, LTBR, MMP-9, and TSLP. The infusion of insulin led to the suppression of IL-4 mRNA expression in MNC starting at $2 \mathrm{~h}$, increasing at $4 \mathrm{~h}$, and maximizing at $6 \mathrm{~h}$ by $44 \pm 7 \%$ $(P<0.05)$ below the baseline, in spite of the cessation of the infusion at $4 \mathrm{~h}$. (Figure 1(a)). ADAM-33 mRNA expression was suppressed significantly by $20 \pm 8 \%$ following insulin infusion $(P<0.05)$ (Figure 1(b)). Additionally, insulin infusion suppressed the expression of LIGHT and LTBR expression by $22 \pm 9 \%$ and $19 \pm 7 \%$ at $4 \mathrm{hr}(P<0.05)$ (Figures $1(\mathrm{c})-1(\mathrm{~d}))$. There was no significant change in the mRNA expression of MCP-1, TSLP, and MMP-9 in MNC following insulin. In the control arms, infused with dextrose alone or saline, there was no alteration in the expression of IL-4, ADAM-33, LIGHT, and LTBR in MNC.

3.3. Effect of Insulin Infusion on Plasma Levels of LIGHT, TGF 1 , NOM, MCP-1, TSLP, and MMP-9. The infusion of insulin in T2DM patients caused a significant suppression of LIGHT concentrations by $25 \pm 5 \%$ (from $39.4 \pm 10.6$ to $27.5 \pm 6.4 \mathrm{pg} / \mathrm{mL}, P<0.05$, Figure 2(a)). Following insulin infusion, plasma concentration of $\mathrm{NOM}\left(\mathrm{NO}_{2} / \mathrm{NO}_{3}\right)$ fell by $34 \pm 15 \%$ at $4 \mathrm{~h}(P<0.05)$ (Figure $2(\mathrm{~b}))$. Additionally the infusion of insulin caused a significant suppression of TGF$\beta 1$ concentrations by $28 \pm 6 \%$ (from $10.8 \pm 1.2$ to $7.7 \pm$ $1.0 \mathrm{ng} / \mathrm{mL}, P<0.05$, Figure $2(\mathrm{c}))$. This suppression occurred in parallel with significant $(P<0.05$, for all) and consistent reductions in ROS generation by MNC (by $18 \pm 5 \%$ ) and serum levels of MCP-1 (by $15 \pm 4 \%$ ) and MMP-9 (by $14 \pm$ $5 \%$ ) at $4 \mathrm{~h}$ following insulin infusion (Table 1). Plasma TSLP concentrations did not alter after insulin (from $10.0 \pm 1.2$ to $10.5 \pm 1.2 \mathrm{pg} / \mathrm{mL}$ at $4 \mathrm{hr}$ ). There was no significant change in NOM, LIGHT concentration, or other inflammatory indices following dextrose or saline infusions.

\section{Discussion}

Our data show for the first time that intravenous infusion of insulin leads to the suppression of IL- 4 expression in MNC at $2 \mathrm{~h}$ by $25 \%$ and by $34 \%$ at $4 \mathrm{~h}$. A further increase in the suppression of IL- 4 by $43 \%$ was evident at $6 \mathrm{~h}$ in spite of the cessation of the infusion at $4 \mathrm{~h}$. Thus the IL- 4 suppressive action of insulin is rapid, potent, and prolonged. This is of interest since a preparation of a monoclonal antibody against the alpha subunit of IL-4 has recently been shown to suppress clinical activity of asthma as reflected in exacerbation rates, $\mathrm{FeV}_{1}$, and mean nocturnal awakening rates [16]. In addition, IL-4 antibody reduced exhaled $\mathrm{NO}$ and plasma eotaxin concentrations. Our current data show that insulin also reduced plasma concentrations of NO metabolites. The fall of NOM in plasma parallels the reduction of NO in exhaled air as discussed below. We have previously shown that insulin suppresses eotaxin concentrations along with other chemokines and chemokine receptors [12], as also discussed below.

Our current data also show that there was a concomitant suppression of ADAM-33, LIGHT, and LTBR, three other mediators known to be associated with the pathogenesis of asthma. These actions are consistent with our previous 


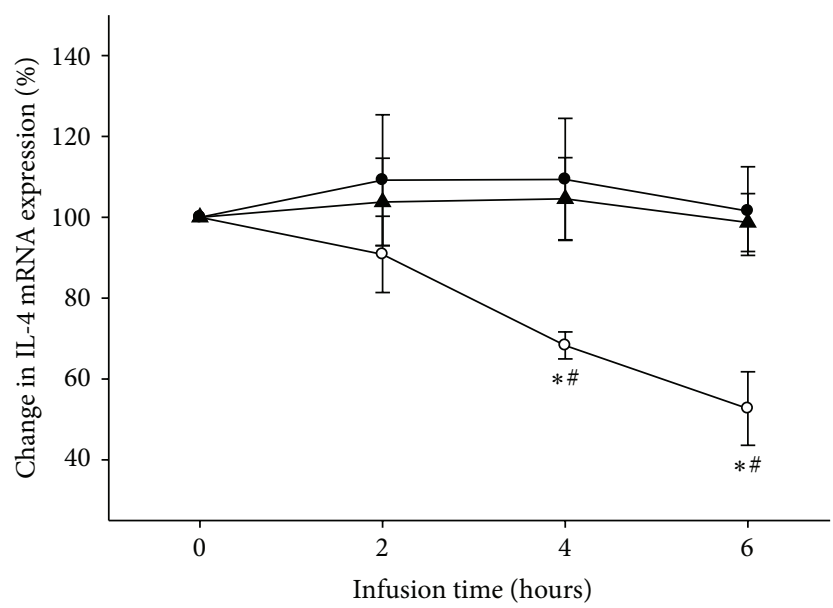

(a)

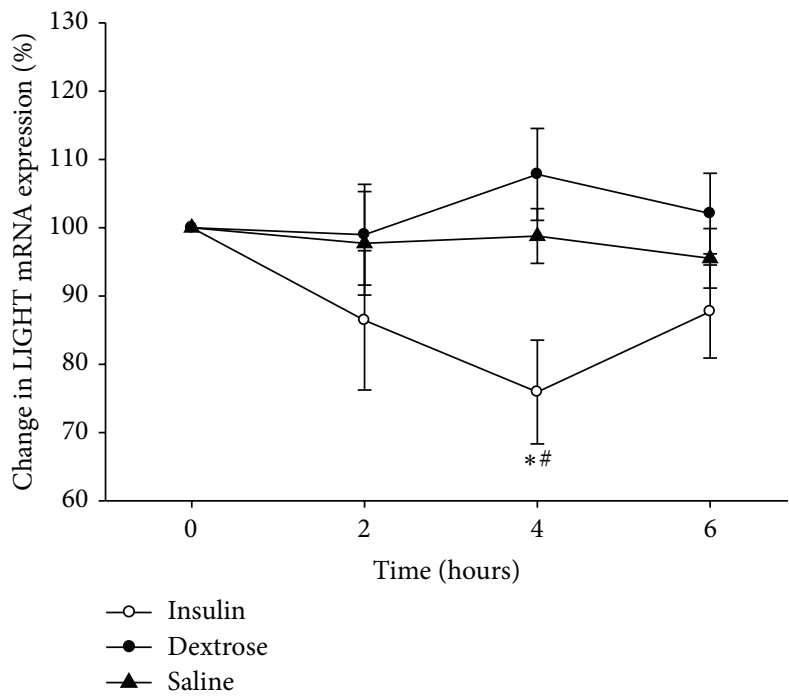

(c)

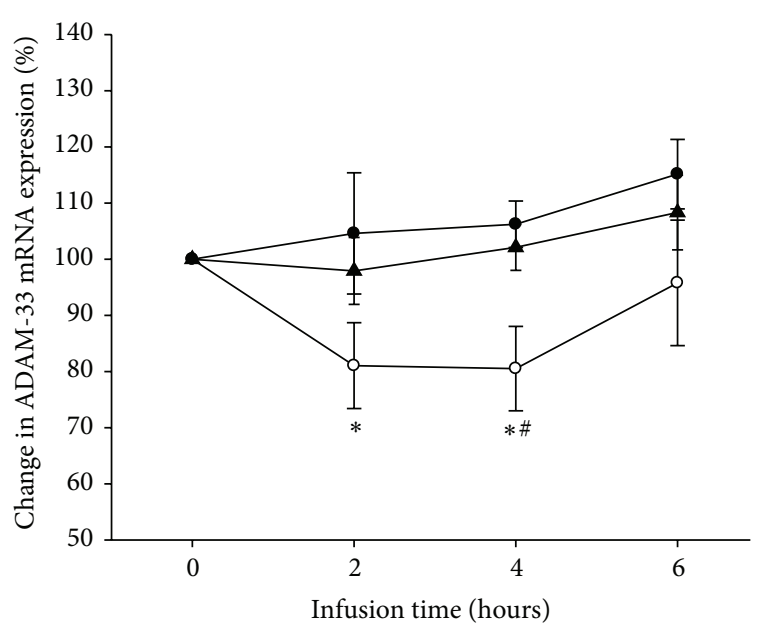

(b)

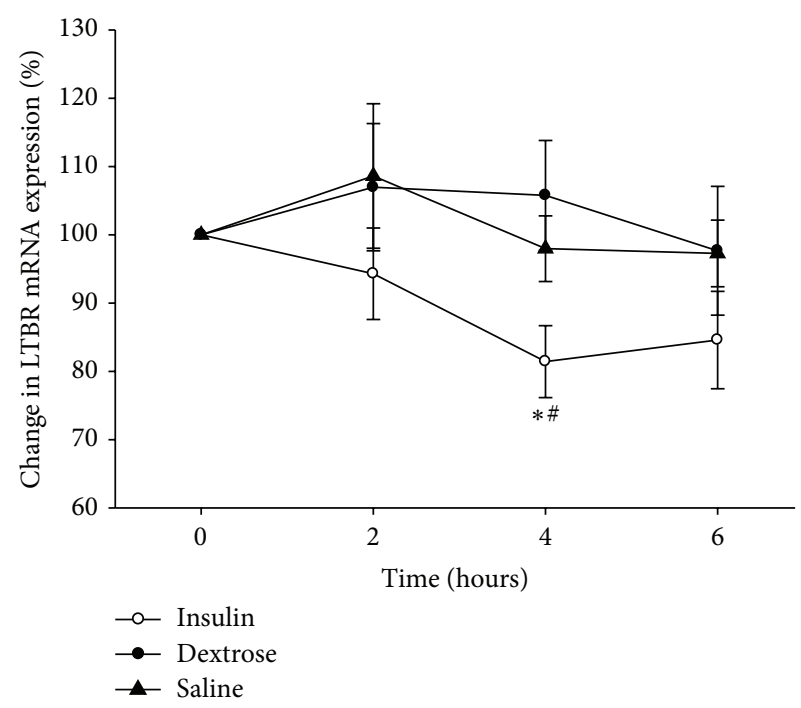

(d)

FIGURE 1: Percent change in (a) IL-4 (b) ADAM-33 (c) LIGHT, and (d) LTBR mRNA expression in MNC following 2 U/hr insulin/dextrose (insulin), dextrose alone (dextrose), or saline alone (saline) infusions for $4 \mathrm{hr}$ in obese T2DM patients. Data is presented as mean \pm SE. ${ }^{*} P<0.05$, when compared to baseline by one-way RMANOVA; ${ }^{*} P<0.05$ compared to control groups by 2 -way RMANOVA.

observations on the anti-inflammatory effects of insulin as reflected in effects like the suppression of intranuclear $\mathrm{NF} \kappa \mathrm{B}$ binding, ROS generation, p $^{\text {phox }}$ expression, Egr-1 binding and expression, and plasma ICAM-1, MCP-1, MMP-9, VEGF, and PAI-1 concentrations $[10,11]$. Insulin also induces an increase in $\mathrm{I} \kappa \mathrm{B} \alpha$ expression, consistent with its ability to suppress $\mathrm{NF} \kappa \mathrm{B}$ binding. There was no change in the expression of TSLP.

The infusion of insulin led to the concomitant suppression of the expression of ADAM-33 and plasma concentrations of MMP-9, both of which are matrix metalloproteinases which participate in the pathogenesis of asthmatic inflammation and bronchial remodeling. LIGHT and its receptor LTBR have very recently been shown to be involved in bronchial remodeling and sensitization to allergens [5]. The concomitant reduction in the cellular expression and plasma concentrations of LIGHT is, therefore, of great interest. Consistent with the suppression of LIGHT and LTBR, there was a reduction in plasma TGF $\beta$ which is responsible for the fibrosis and remodeling through epithelial-mesenchymal transformation [17]. There was also a significant suppression of plasma concentrations of MCP-1 (CCL-2). Our previously published data also demonstrate that plasma concentrations of RANTES (CCL-5) and eotaxin (CCL-11) and the expression of chemokine receptors CCR- 2 and CCR- 5 are also suppressed by an insulin infusion [12]. Chemokines attract monocytes and eosinophils and thus actively participate in the pathogenesis of allergic asthmatic inflammation. There was, however, no change in plasma TSLP concentrations.

Our data also show that insulin infusion suppresses the plasma concentration of NOM, suggestive of a reduction in NO generation, probably from iNOS. This observation is consistent with a previous demonstration that, in patients in intensive care, the infusion of insulin leads to a reduction 


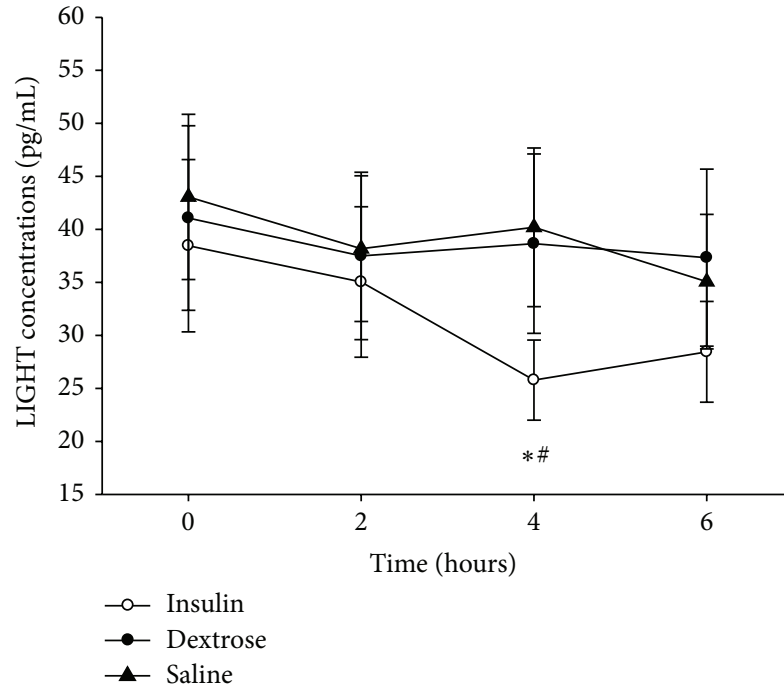

(a)

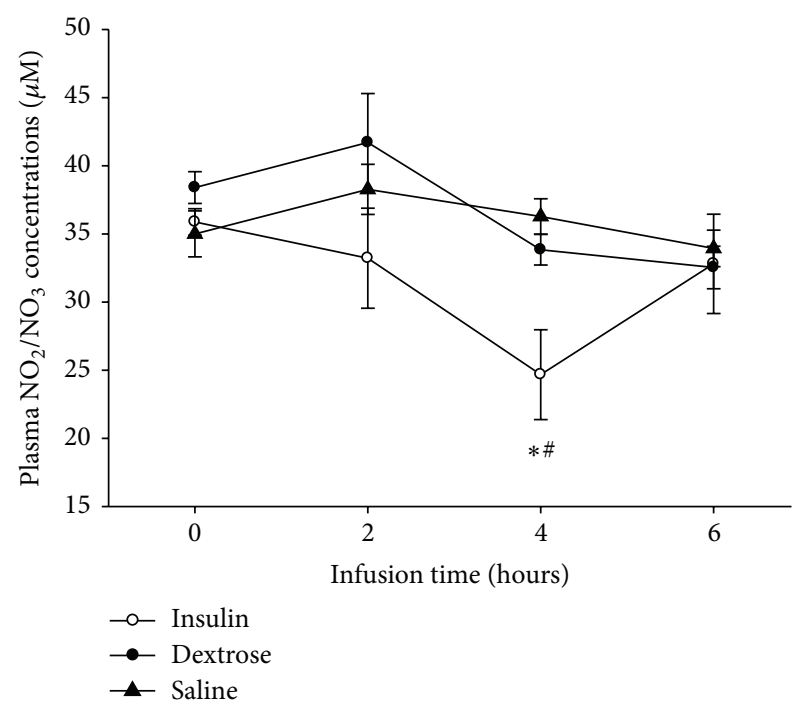

(b)

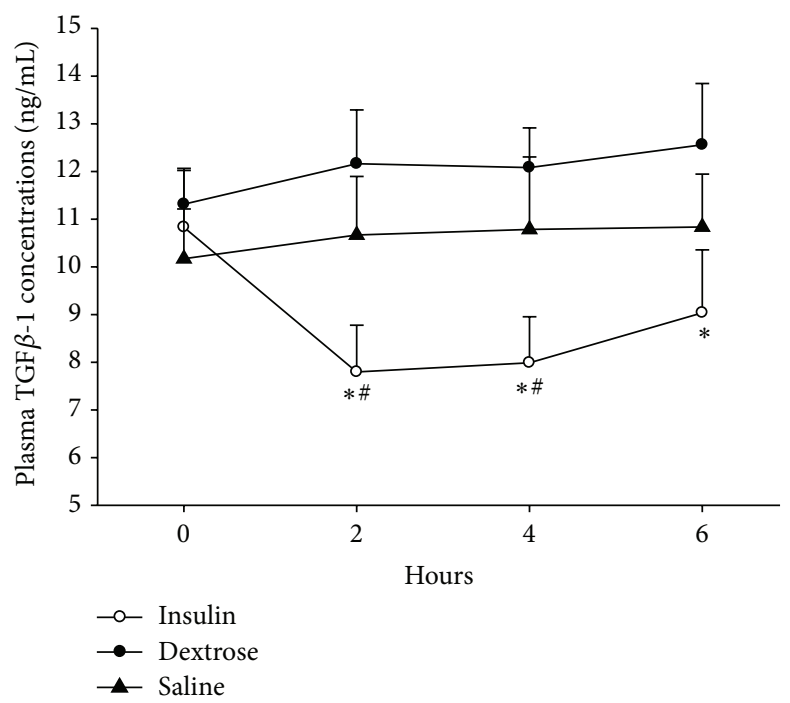

(c)

Figure 2: Change in (a) LIGHT, (b) NOM, and (c) TGF- $\beta 1$ concentrations following $2 \mathrm{U} / \mathrm{hr}$ insulin/dextrose (insulin), dextrose alone (dextrose), or saline alone (saline) infusions for $4 \mathrm{hr}$ in obese T2DM patients. Data is presented as mean $\pm \mathrm{SE}$. ${ }^{*} P<0.05$, when compared to baseline by one-way RMANOVA; ${ }^{*} P<0.05$ compared to control groups by 2 -way RMANOVA.

in plasma concentration of NOM with a concomitant suppression of iNOS expression in the liver [18]. We have recently observed that the rapid increase in NO metabolites in plasma following the injection of endotoxin in normal subjects [19], indicative of a marked stimulation of iNOS from macrophages in the reticuloendothelial system, is totally inhibited by insulin given at a dose similar to that used in the current study. Since NO in exhaled air is considered to be indicative of intrabronchial inflammation in asthma and since this NO is generated by iNOS in bronchial macrophages [20], the suppression of NOM by insulin in our study suggests that insulin may also possibly be able to suppress NO generation by iNOS in bronchial macrophages and NO in exhaled air. ROS generation by MNC was also reduced by insulin infusion consistent with our previous observations [11]. It has also been shown previously that hydrocortisone has an ROS suppressive effect [21]. Furthermore, our recent data show that an injection of $300 \mathrm{mg}$ of hydrocortisone $(=60 \mathrm{mg}$ prednisone) suppresses the expression of IL-4, LTBR, ADAM33, and MMP-9 and the plasma concentration of MCP-1. The magnitude of this effect is similar to that of insulin infusion described in this study (unpublished observations). On the other hand, we have recently observed several paradoxical proinflammatory effects of this dose of hydrocortisone including an increase in plasma MMP-9 and HMG-B1 concentrations in parallel with increases in plasma glucose and FFA concentrations [22], all of which are suppressed by insulin. These data suggest that a combination of insulin and corticosteroids may form a future potential therapy for asthma. 
The potential clinical significance of our observations is readily apparent. The insulin resistant proinflammatory states of obesity and type 2 diabetes are characterized by an increase in IL-4, MMP-9, LIGHT, and CCR-2 expression in MNC and MMP-9 and NOM concentrations in plasma [1]. Since IL-4, LIGHT, LTBR, TGF $\beta$, MMP-9, and NO are key mediators involved in the pathogenesis of allergy and asthma, the increase in their expression may contribute to the increased vulnerability and risk of asthma in the obese. It is relevant that weight reduction following gastric bypass surgery leads to a reversal of these increases in parallel with the restoration of insulin sensitivity [1]. By the fact that insulin infusion suppresses the expression of IL-4, ADAM-33, LIGHT, and LTBR and the plasma concentrations of NOM and MMP-9, the efficacy of insulin in the treatment of asthma needs to be assessed. In addition, insulin has been shown to suppress the plasma concentrations of several other relevant chemokines like RANTES (CCL-5) and the expression of their receptors like CCR-5 [12]. Its action may be additive to that of corticosteroids and may potentially reduce the dose of corticosteroids that is used to treat exacerbations of asthma in hospitalized patients. It is also relevant that insulin suppresses TLR2 and TLR-4 [13], which mediate inflammatory responses in response to Gram positive bacteria and LPS, a product of Gram negative bacteria, respectively. In addition, it also suppresses the expression of TLR-7 and TLR-9 which get activated by RNA and DNA containing viruses, respectively [13]. There is evidence that these pattern recognition receptors contribute to the pathogenesis of asthma [23].

The fact that insulin resistant states lead to an increase in the expression of asthma related genes in spite of accompanying hyperinsulinemia and the fact that a low dose insulin infusion leads to the suppression of these genes need to be explained. The pathogenesis of insulin resistance is now thought to be due to an increase in proinflammatory genes [24] and free fatty acid (FFA) concentrations [25]. FFAs have also been shown to be proinflammatory [26]. These proinflammatory genes and FFAs interfere with insulin signal transduction $[24,27]$. The increase in obesity related increase in asthma related genes is probably the result of systemic inflammation which concomitantly induces insulin resistance and hyperinsulinemia. However, this increase in insulin is not sufficient to suppress the inflammatory response. Insulin concentrations need to be increased beyond these concentrations by 3 to 4 times to induce an anti-inflammatory effect, be it the suppression of $\mathrm{NF} \kappa \mathrm{B}$ or other inflammatory factors like chemokines or asthma related genes. Thus, the plasma concentrations of insulin need to overwhelm the resistance posed by the interference in insulin signaling caused by inflammation and FFAs.

There is an important limitation to this study since our investigations did not include patients with asthma and thus the observations cannot be applied to asthmatics immediately. However, they demonstrate for the first time the ability of insulin to suppress several of these key mediators following a short period of infusion in a group of patients with an increased risk of asthma. Clearly, a study investigating the effect of intravenous infusion on respiratory function in asthmatic patients needs to be carried out and is currently being planned at our center.

In conclusion, in patients with obesity and type 2 diabetes, insulin infusion leads to a suppression of the expression of IL4, ADAM-33, LIGHT, and LTBR in parallel with a reduction in plasma NOM, TGF $\beta$, MCP-1, and MMP-9 concentrations. The potential role of intravenous infusion of insulin in the treatment of asthmatic patients needs to be investigated.

\section{Conflict of Interests}

The authors declare that there is no conflict of interests regarding the publication of this paper.

\section{Acknowledgments}

Paresh Dandona is supported by grants from NIH (R01DK092653 and R01-DK075877), Juvenile Diabetes Research Foundation (172013267), and the American Diabetes Association (112CT20). Paresh Dandona also has support from Merck, Novo Nordisk, Boehringer Ingelheim, and AbbVie Pharmaceuticals. The authors are grateful to Lynne Barnas for secretarial support.

\section{References}

[1] P. Dandona, H. Ghanim, S. V. Monte et al., "Increase in the mediators of asthma in obesity and obesity with type 2 diabetes: Reduction with weight loss," Obesity, vol. 22, no. 2, pp. 356-362, 2014.

[2] C. Doucet, D. Brouty-Boyé, C. Pottin-Clémenceau, G. W. Canonica, C. Jasmin, and B. Azzarone, "Interleukin (IL) 4 and IL-13 act on human lung fibroblasts. Implication in asthma," The Journal of Clinical Investigation, vol. 101, no. 10, pp. 2129-2139, 1998.

[3] I. C. J. de Faria, E. J. de Faria, A. A. D. C. Toro, J. D. Ribeiro, and C. S. Bertuzzo, "Association of TGF- $\beta 1$, CD14, IL-4, IL-4R and ADAM33 gene polymorphisms with asthma severity in children and adolescents," Jornal de Pediatria, vol. 84, no. 3, pp. 203-210, 2008.

[4] S. Romagnani, "Cytokines and chemoattractants in allergic inflammation," Molecular Immunology, vol. 38, no. 12-13, pp. 881-885, 2002.

[5] T. A. Doherty, P. Soroosh, N. Khorram et al., "The tumor necrosis factor family member LIGHT is a target for asthmatic airway remodeling," Nature Medicine, vol. 17, no. 5, pp. 596-603, 2011.

[6] S.-E. Dahlén, "TSLP in asthma-a new kid on the block?" The New England Journal of Medicine, vol. 370, no. 22, pp. 2144-2145, 2014.

[7] S. A. Kharitonov and P. J. Barnes, "Effects of corticosteroids on noninvasive biomarkers of inflammation in asthma and chronic obstructive pulmonary disease," Proceedings of the American Thoracic Society, vol. 1, no. 3, pp. 191-199, 2004.

[8] S. J. Szefler, H. Mitchell, C. A. Sorkness et al., "Management of asthma based on exhaled nitric oxide in addition to guidelinebased treatment for inner-city adolescents and young adults: a randomised controlled trial," The Lancet, vol. 372, no. 9643, pp. 1065-1072, 2008.

[9] M. Barreto, M. P. Villa, C. Olita, S. Martella, G. Ciabattoni, and P. Montuschi, "8-Isoprostane in exhaled breath condensate and 
exercise-induced bronchoconstriction in asthmatic children and adolescents," Chest, vol. 135, no. 1, pp. 66-73, 2009.

[10] A. Aljada, H. Ghanim, P. Mohanty, N. Kapur, and P. Dandona, "Insulin inhibits the pro-inflammatory transcription factor early growth response gene-1 (Egr)-1 expression in mononuclear cells (MNC) and reduces plasma tissue factor (TF) and plasminogen activator inhibitor-1 (PAI-1) concentrations," Journal of Clinical Endocrinology and Metabolism, vol. 87, no. 3, pp. 1419-1422, 2002.

[11] P. Dandona, A. Aljada, P. Mohanty et al., "Insulin inhibits intranuclear nuclear factor kappaB and stimulates IkappaB in mononuclear cells in obese subjects: evidence for an antiinflammatory effect?" Journal of Clinical Endocrinology and Metabolism, vol. 86, no. 7, pp. 3257-3265, 2001.

[12] H. Ghanim, K. Korzeniewski, C. L. Sia et al., "Suppressive effect of insulin infusion on chemokines and chemokine receptors," Diabetes Care, vol. 33, no. 5, pp. 1103-1108, 2010.

[13] H. Ghanim, P. Mohanty, R. Deopurkar et al., "Acute modulation of toll-like receptors by insulin," Diabetes Care, vol. 31, no. 9, pp. 1827-1831, 2008.

[14] K. Chen, Y. Xiang, X. Yao et al., "The active contribution of Tolllike receptors to allergic airway inflammation," International Immunopharmacology, vol. 11, no. 10, pp. 1391-1398, 2011.

[15] H. Ghanim, P. Mohanty, R. Pathak, A. Chaudhuri, L. S. Chang, and P. Dandona, "Orange juice or fructose intake does not induce oxidative and inflammatory response," Diabetes Care, vol. 30, no. 6, pp. 1406-1411, 2007.

[16] S. Wenzel, L. Ford, D. Pearlman et al., "Dupilumab in persistent asthma with elevated eosinophil levels," The New England Journal of Medicine, vol. 368, no. 26, pp. 2455-2466, 2013.

[17] M. Zhang, Z. Zhang, H.-Y. Pan, D.-X. Wang, Z.-T. Deng, and X.-L. Ye, "TGF- $\beta 1$ induces human bronchial epithelial cell-tomesenchymal transition in vitro," Lung, vol. 187, no. 3, pp. 187194, 2009.

[18] L. Langouche, I. Vanhorebeek, D. Vlasselaers et al., "Intensive insulin therapy protects the endothelium of critically ill patients," Journal of Clinical Investigation, vol. 115, no. 8, pp. 2277-2286, 2005.

[19] P. Dandona, H. Ghanim, A. Bandyopadhyay et al., "Insulin suppresses endotoxin-induced oxidative, nitrosative, and inflammatory stress in humans," Diabetes Care, vol. 33, no. 11, pp. 24162423, 2010.

[20] D. H. Yates, "Role of exhaled nitric oxide in asthma," Immunology and Cell Biology, vol. 79, no. 2, pp. 178-190, 2001.

[21] A. Aljada, H. Ghanim, E. Assian et al., "Increased I $\kappa$ B expression and diminished nuclear NF- $\kappa \mathrm{B}$ in human mononuclear cells following hydrocortisone injection," Journal of Clinical Endocrinology and Metabolism, vol. 84, no. 9, pp. 3386-3389, 1999.

[22] P. Dandona, H. Ghanim, C. L. Sia et al., "A mixed antiinflammatory and pro-inflammatory response associated with a high dose of corticosteroids," Current Molecular Medicine, vol. 14, no. 6, pp. 793-801, 2014.

[23] G. F. G. Bezemer, S. Sagar, J. van Bergenhenegouwen et al., "Dual role of toll-like receptors in asthma and chronic obstructive pulmonary disease," Pharmacological Reviews, vol. 64, no. 2, pp. 337-358, 2012.

[24] G. S. Hotamisligil, "Inflammation and metabolic disorders," Nature, vol. 444, no. 7121, pp. 860-867, 2006.

[25] G. Boden, P. She, M. Mozzoli et al., "Free fatty acids produce insulin resistance and activate the proinflammatory nuclear factor- $\kappa \mathrm{b}$ pathway in rat liver," Diabetes, vol. 54 , no. 12 , pp. $3458-$ 3465, 2005.

[26] D. Tripathy, P. Mohanty, S. Dhindsa et al., "Elevation of free fatty acids induces inflammation and impairs vascular reactivity in healthy subjects," Diabetes, vol. 52, no. 12, pp. 2882-2887, 2003.

[27] K. A. Schulman, B. D. Richman, and R. E. Herzlinger, "Shifting toward defined contributions-predicting the effects," The New England Journal of Medicine, vol. 370, no. 26, pp. 2462-2465, 2014. 


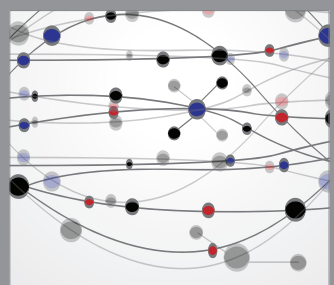

The Scientific World Journal
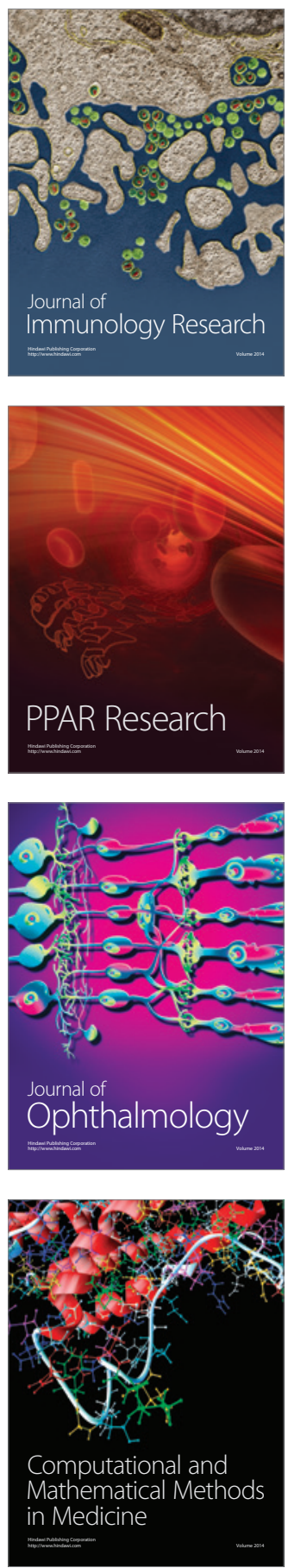

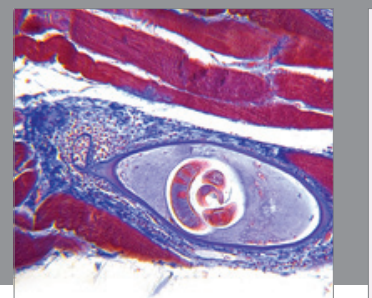

Gastroenterology

Research and Practice
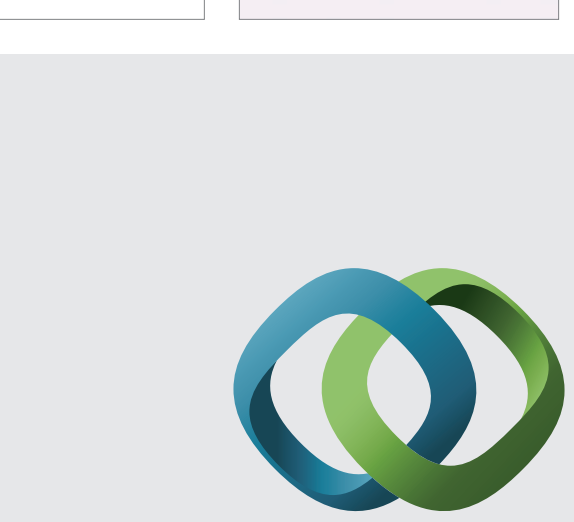

\section{Hindawi}

Submit your manuscripts at

http://www.hindawi.com
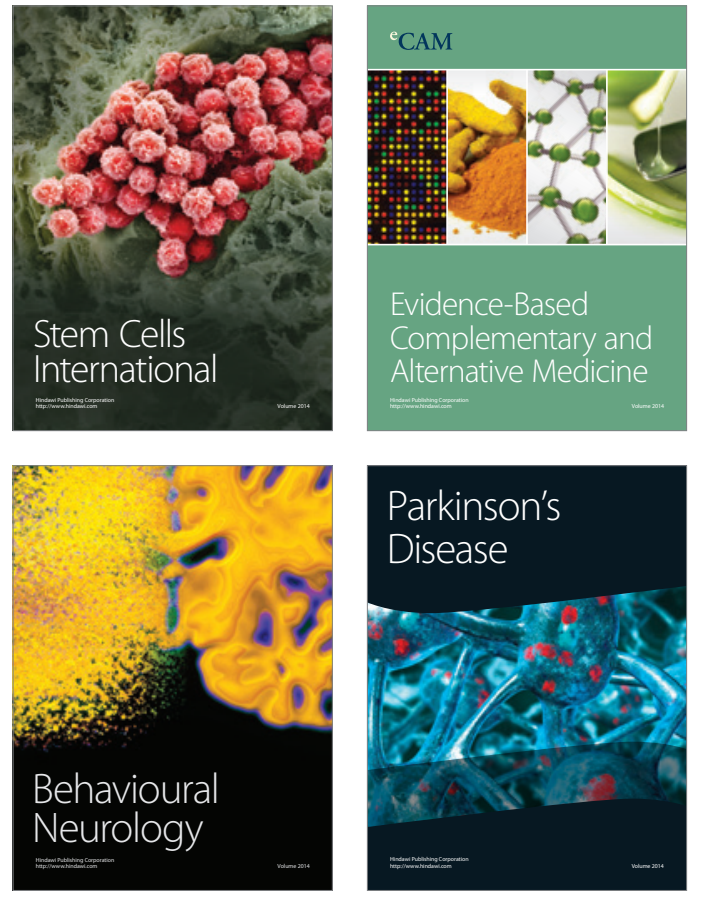
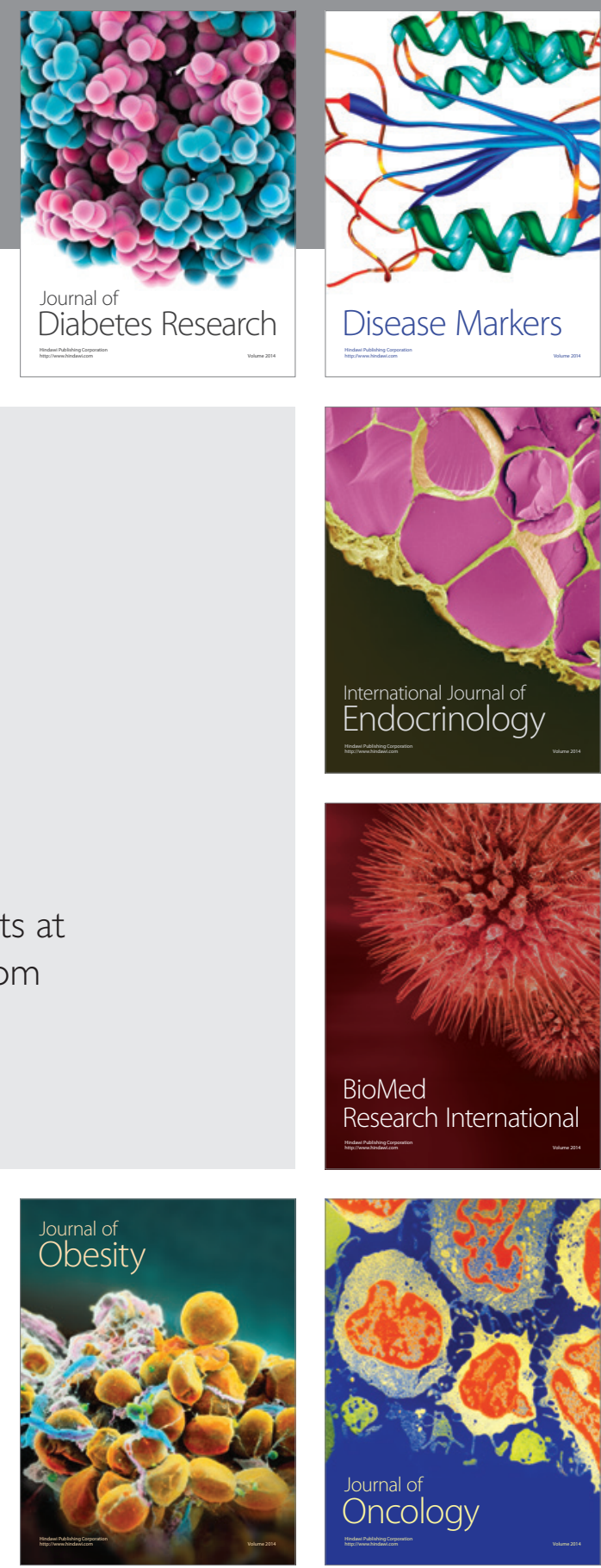

Disease Markers
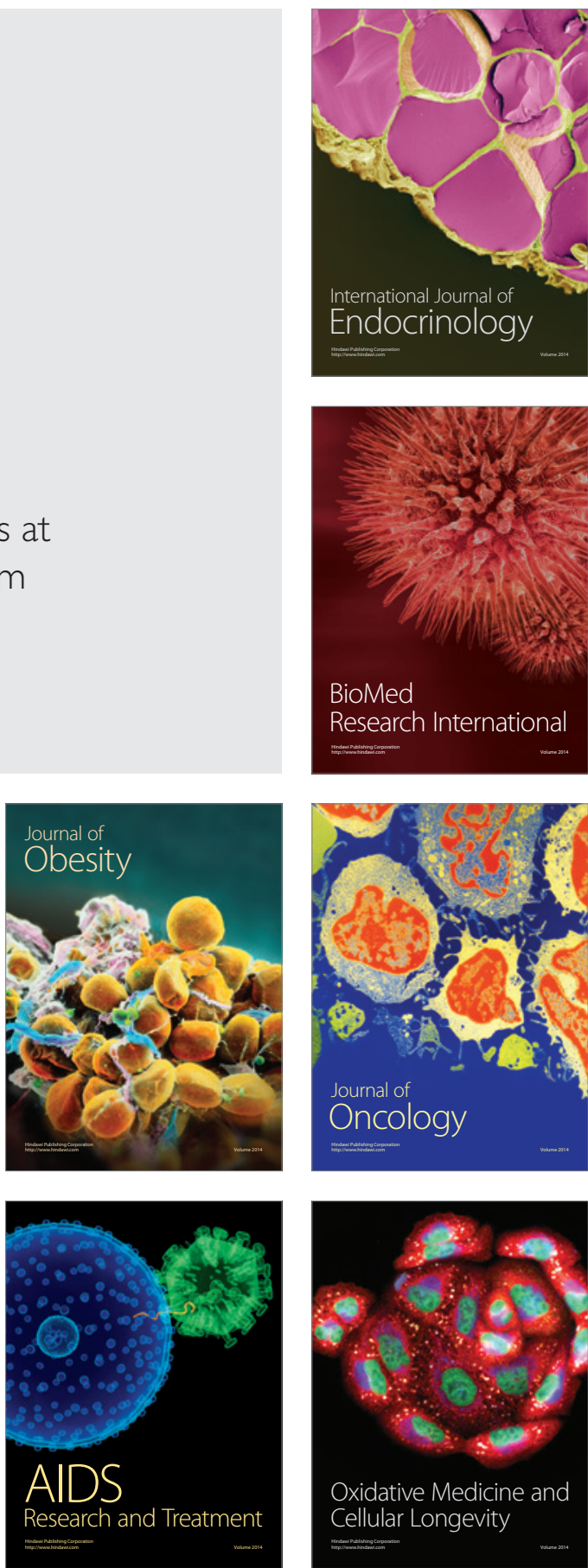